\title{
Counterfactuals vs. Conceivability as a Guide to Modal Knowledge
}

abstract

I compare two prominent approaches to knowledge of metaphysical modality, the more traditional approach via conceiving viz. imagining a scenario and a more recent approach via counterfactual reasoning. In particular, Timothy Williamson has claimed that the proper context for a modal exercise of imagination is a counterfactual supposition. I critically assess this claim, arguing that a purely conceivability/imaginability-based approach has a key advantage compared to a counterfactual-based one. It can take on board Williamson's insights about the structure of modal imagination while avoiding aspects of counterfactual reasoning which are orthogonal to figuring out metaphysical modality. In assessing whether $A$ is possible, we creatively devise test scenarios, psychologically and metaphysically apt $A$-scenarios, which manifest the relevant metaphysical requirements and test them for their compatibility with $A$. In this exercise, imagination is subject to implicit constraints as Williamson has it, but it is not bound to drawing consequences from minimally altering actuality such as to make room for $A$.

I shall compare two views which even their detractors often regard as 'the leading current accounts of modal epistemology: the conceivability and counterfactual accounts.'(Jago forthcoming) I focus on questions of possibility and leave necessity for future work. I shall only discuss the most prominent version of the counterfactual-based account due to Timothy 
Williamson (2007). ${ }^{1}$ As contrasted to 'counterfactual-based', I reserve 'conceivability-based' for an approach in which the specific counterfactuals Williamson has in mind do not play a distinctive role. I use 'imagination-deploying' to address the conceivability- and the counterfactual-based account together.

In concentrating on Williamson, I limit the scope of my argument by making a presupposition that is shared by Williamson and proponents of conceivability-based approaches like Yablo $(1993,2)$ and Chalmers (2002): the task is to figure out a canonical (unique and general) method which allows us to attain any knowledge of metaphysical possibility within human reach. The presupposition may be made explicit: if there is a canonical method, what is the most promising candidate? The availability of such a canonical method is compatible with there being other, less comprehensive methods to arrive at modal claims.

I shall not discuss theories like Kment's (2014, forthcoming), who assigns counterfactuals a limited role in proceeding from one possibility claim to another. My argument does not preclude such a limited use of counterfactuals in settling certain modal claims. Although I compare counterfactual-based and conceivability-based approaches with regard to the canonical method, I am also open to a pluralistic or non-uniform approach: there is no canonical method. Different methods, among them counterfactual reasoning and imagination, may be put to different uses in modal reasoning. I shall briefly indicate how imagination may be integrated into a pluralistic approach.

Imagination-deploying modal epistemologies have been intensely discussed. Interesting alternatives have recently emerged (some examples in Fischer and Leon 2017). Thus, my project

\footnotetext{
1 Other versions are Lange (2005), Hill (2006), Yli-Vakkuri (2013), Kroedel (2012, 2017), Kment (2014), forthcoming.
} 
may seem obsolete. I shall therefore start with some motivational considerations, identifying the gap my paper is to close. ${ }^{2}$ In trying to close this gap, I shall also give some hints why I do not reckon the main criticisms of imagination-deploying epistemology fatal.

Williamson offers the counterfactual-based approach as a friendly amendment to conceivability-based ones. Williamson's amendment has three advantages. First, many of the founding texts that shaped the present debate suggest a connection between using imagination and counterfactuals in modal considerations. Philosophers like Kripke (1980), Yablo (1993), and Chalmers (2002) frame (part of) their exercises of modal imagination as dwelling on counterfactual situations. However, the relationship between imagination and counterfactual reasoning needs clarification. Williamson's amendment provides such a clarification.

Second, conceivability-based modal epistemology has come under pressure from several sides. It is usually associated with modal rationalism or 'neo-rationalism', ${ }^{3}$ indicating that it heavily relies on the use of 'higher' cognitive capacities and resources like conceptual and $a$ priori knowledge. Philosophy is exempt from other disciplines in its special reliance on these capacities, to the dismay of naturalistically-minded critics (e.g. Martínez 2015, Bueno and Shalkowski 2015). Williamson's view is anti-exceptionalist: imagination does not recruit any capacities beyond those required anyway by assessing counterfactuals in everyday life. Hence a conceivability-based account that takes inspiration from Williamson's anti-exceptionalism is an interesting alternative to (but does not exclude) modal rationalism.

\footnotetext{
${ }^{2}$ Thanks to an anonymous referee for raising the issue.

${ }^{3}$ Martínez (2015, 659), targeting positions like Yablo (1993), Peacocke (1999), Chalmers (2002), Ichikawa and Jarvis (2012).
} 
Third, there are long-standing doubts as to how far imagination can be properly restrained to metaphysical possibility (cf. Tidman 1994, Byrne 2007, Fiocco 2007, Berto and Schoonen 2018, Jago forthcoming). For instance, Yablo says that I cannot modally imagine Goldbach's Conjecture (GB) to be true/false $(1993,39)$. But I arguably can imagine GB to be true/false by imagining a prize ceremony in which I am awarded the Fields medal for having proven/refuted GB (Kung 2016, 96, Priest 2017). Nothing follows for the possible truth or falsity of GB. For imagining to provide modal knowledge, it must be a certain kind of imagining (Yablo 1993, 26). Modal imagining properly responds to a modal issue. Williamson's counterfactual-based approach makes good on this claim, which otherwise might seem ad hoc:

'Discussions of the epistemology of modality often focus on imaginability or conceivability as a test of possibility while ignoring the role of the imagination in the assessment of mundane counterfactuals. In doing so, they omit the appropriate context for understanding the relation between modality and the imagination... Imagination can be made to look cognitively worthless. Once we recall its fallible but vital role in evaluating counterfactual conditionals, we should be more open to the idea that it plays such a role in evaluating claims of possibility and necessity.'(Williamson 2007, 163)

I have summarized three advantages of Williamson's specific claim that the conceivability-based approach should be reformulated as a counterfactual-based one. However, doubts remain: does the modal use of imagination really boil down to counterfactual reasoning as Williamson has it, or does imagination have an independent function in acquiring modal knowledge which is not captured and perhaps even missed by the counterfactual account? To my 
knowledge, this particular question has not been addressed so far. ${ }^{4}$ Discussing it not only closes a gap in current debate, it also allows me to fine-tune the contrast of the two options of a conceivability-based and a counterfactual-based approach in a way that manifests the key advantage of the former. I shall argue for the following claims: imagination plays an independent role in modal reasoning, which may be tentatively characterized as creative imaginative experimenting. This role is not captured by the extant counterfactual-based account. Nevertheless, a purely conceivability-based account can take on board Williamson's main lessons on the proper use of imagination. I do not claim that we cannot, or that we never do proceed in the way indicated by Williamson. Yet since proceeding in this way depends on a use of imagination that justifies modal claims independently of counterfactual reasoning, the latter becomes an unnecessary detour.

I summarize the core argument to be developed:

For any candidate for possibility $A$ :

(i) Let $M$ be the canonical method of acquiring modal knowledge whether $A$ is possible and $I$ be the issue whether a contradiction is true at the closest $A$-scenarios.

(ii) According to the counterfactual-based account, addressing $I$ is part of $M$.

(iii) The canonical method $\mathrm{M}$ is coherent imagining.

(iv) Addressing $I$ is not part of coherent imagining.

(v) The counterfactual-based account is false.

\footnotetext{
${ }^{4}$ Gregory $(2004,2017)$ criticizes the counterfactual-based account and endorses an independent conceivability-based one, but he does not directly address their relationship.
} 
I give an overview of the considerations to come: (1.) I present the counterfactual approach. (1.1.) I summarize Williamson's picture. (1.2.) I argue that modal reasoning is often psychologically immediate, (1.2.1.) drawing on tacit modal constraints and (1.2.2.) cognitive dissonance as an operational criterion of impossibility. (1.3.) I describe how imaginative experiments work as a dissonance test. (1.3.1.) I give a differential diagnosis of dissonance. (1.3.2.) I discuss the use of imaginative experiments in manifesting and testing modal constraints. (2.) I argue that the resulting use of imagination dispenses with counterfactual reasoning. (2.1.) I make my case by examples. (2.2.) I declare the autonomy of coherent imagining. (2.3.) I anticipate objections. (2.4.) I draw an illustrative parallel to standard thought-experiments. (3.) I close with two lessons for modal imagination.

\section{The Counterfactual Approach to Modal Conceiving}

\subsection{Williamson's Picture}

I give an opinionated survey of Williamson's account. A regimented use of imagination leads from testing the counterfactual supposition that A for coherence to a possibility claim. The imaginative development has to cover relevant A-scenarios in relevant detail. It is subject to conditions like staying maximally close to actuality.

Williamson uses the following logical equivalences:

$$
\begin{aligned}
& \diamond \mathrm{A} \equiv \neg(\mathrm{A} \square \rightarrow \perp) \\
& \square \mathrm{A} \equiv(\neg \mathrm{A} \square \rightarrow \perp)
\end{aligned}
$$


Williamson presents his use of these equivalences as a conceivability test:

'by [the equivalences], we assert $\diamond \mathbf{A}$ when our counterfactual development of the supposition $\mathbf{A}$ does not robustly yield a contradiction (and we do not attribute the failure to a defect in our search); we deny $\diamond \mathbf{A}$ when our counterfactual development of $\mathbf{A}$ robustly yields a contradiction. Thus our fallible imaginative evaluation of counterfactuals has a conceivability test for possibility and an inconceivability test for impossibility built in as fallible special cases.'(Williamson 2007, 163)

Williamson's proposal continues a tradition of interpreting the modal use of imagination as a coherence test: we are justified to judge that $A$ is possible if we can coherently imagine an $A$ scenario in sufficient detail (cf. Chalmers 2002, 153, Ichikawa and Jarvis 2012, 131). However, as we shall see, the required notion of coherence is quite strong. It demands compatibility with 'constitutive matters' like the atom number of gold being 79. Williamson claims that a counterfactual version of the coherence test yields precisely the right notion of coherence: we may justifiedly albeit defeasibly judge that $A$ is possible if we can develop the counterfactual supposition of $A$ in imagination coherently and in relevant detail, i.e. without robustly encountering a contradiction, and that $A$ is impossible if we cannot so develop it. Williamson's test depends on the vacuous truth of counterfactuals with a contradiction in the consequent $(\ldots \square \rightarrow \perp$, henceforth $C C)$ and an impossible antecedent. ${ }^{5}$

\footnotetext{
5 The alternative is that there are non-vacuous counterpossibles (cf. Berto et al. 2018, Jago forthcoming). It may be a further advantage of a conceivability-based account that the alternative does not have to bother us.
} 
In the rest of this section, I list further relevant features of the account. Imagination is used in a highly regimented way. It can recruit any information and any mental capacity 'offline', for instance in assessing a mundane counterfactual like 'if the bush had not been there, the rock would have ended in the lake':

'your imaginative exercise is radically informed and disciplined by your perception of the slope and the rock and your sense of how nature works. Thus the imagination can in principle exploit all our background knowledge in evaluating counterfactuals.'(Williamson 2007, 143)

I assume this view of imagination to be common ground between the imagination-deploying approaches under discussion.

Williamson's condition of robustness needs commenting. Williamson says:

'In fact, if one finds various different ways of imagining the antecedent equally good, one may try developing several of them, to test whether they all yield the consequent.'(Williamson 2007, 153)

Our elaboration should cover a certain range of possible variations of the antecedent scenario. The question becomes which ways of imagining the antecedent we ought to pursue. As we shall see, this is a crucial question; it uncovers a substantial difference between the counterfactualbased and a plausible conceivability-based approach.

A related issue is when the antecedent scenario is sufficiently developed (cf. Strohminger and Yli-Vakkuri 2018, 317). The issue also plagues other approaches to conceivability. One has to suitably flesh out a scenario (cf. Chalmers 2002, 149-150). The resulting challenge can be 
illustrated by examples of van Inwagen's $(1998,71,78)$ : could there be a naturally purple cow? Could there be transparent iron? In order to answer these questions, van Inwagen suggests, we would have to imagine molecular deep structures that would make cows naturally purple and iron transparent. The problem spreads to more mundane possibilities like the possibility of a completely noise-free vinyl record player (Geirsson 2005, 287).

Williamson indirectly provides guidance to solving this issue in discussing when we have reasons to deny a counterfactual:

'...if [the consequent] has not emerged after a given period of development the question remains whether it will emerge in the course of further development, for lines of reasoning can be continued indefinitely from any given premise. To reach a negative conclusion, one must in effect judge that if the consequent were ever going to emerge it would have done so by now... But we are capable of making such assessments.'(Williamson 2007, 153-155)

This quote harbours an answer when a counterfactual has been sufficiently developed to support a possibility claim: we implicitly judge that, if the contradiction were ever going to emerge, it would have done so by now.

I highlight a facet of the challenge. The issue is not only when a scenario is specific enough, but also when it is relevantly specified. We must be guided by a sense for those details which are relevant to detecting a contradiction. In sum, we have to pursue relevant ways of developing the antecedent in relevant detail.

The use of a counterfactual supposition comes with certain semantic requirements. There are many approaches to the semantics of counterfactuals. I shall make do with the near-standard 
condition of minimum alteration, to which Williamson himself subscribes:

'We seem to have a pre-reflective tendency to minimum alteration in imagining counterfactual alternatives to actuality, reminiscent of the role that similarity between possible worlds plays in the Lewis-Stalnaker semantics.'(Williamson 2007, 151)

I shall base my criticism of the counterfactual-based approach on the semantic requirement of minimal alteration. If this requirement is replaced by a different semantics, it has to be seen whether my criticism still applies.

Williamson suggests that imagination by default proceeds in accordance with counterfactual reasoning, in particular the requirement of minimally altering reality:

"The default for the imagination in its primary function may be to proceed as "realistically" as it can, subject to whatever deviations the thinker imposes by brute force...'(Williamson 2007, 142)

If imagination is by default properly constrained, its modal function does not depend on explicitly framing its exercise as counterfactual reasoning. I shall exploit this independence to argue that the use of imagination can stand on its own.

\subsection{The Immediacy of Modal Imagination}

\subsubsection{Tacit Modal Constraints}

I use two objections from the literature to qualify the account: we have to develop the supposition of $A$ in a way to which our everyday methods of assessing counterfactuals apply. The 
metaphysical constraints that bear on this development must be largely implicit in a psychologically immediate reasoning process.

The first criticism of the counterfactual-based account is the following: Williamson's modal epistemology is supposed to be anti-exceptionalist. But our methods of assessing everyday counterfactuals cannot be transferred to Williamson's test for possibility (Gregory 2017). They do not generally apply to any $C C$. Consider:

(GOLD) Gold could not have had an atom number different from 79.

(GOLD) can be inferred from:

(GOLDC) If gold had an atom number different from 79, 2+2 would be 5 .

Yet how do we know GOLDC? Natural ways of knowing GOLDC draw on knowledge that the antecedent is impossible or on deriving GOLDC from some other CC with the same antecedent. Known in this way, GOLDC does not really contribute to our knowing GOLD. There are doubts as to whether there are ways of knowing GOLDC which better suit Williamson's task.

In reply, Williamson's procedure should not be described in one of the following ways: a) We suppose that $A$ and randomly reason along until we perchance encounter a contradiction. b) 
We consider an arbitrarily chosen contradiction in the consequent. ${ }^{6}$ Instead, we should develop the counterfactual supposition with an eye on finding and assessing relevant $C C$. Our everyday methods may not apply to $C C$ in general, but Williamson has a story about how they apply to relevant $C C$ like the following:

(GOLDCC) If gold had an atom number different from 79, it would have an atom number different from 79 and it would have atom number 79.

To appreciate Williamson's view of how we know GOLDCC, I turn to the second criticism. The criticism is that imagination-deploying accounts presuppose something too close to explicit modal knowledge. For us to know GOLDCC, the actual atom number of gold 79 has to be imported into the supposed scenario although this conflicts with supposing that the atom number is different. A danger of circularity arises if we have to knowledgeably discern necessary constraints like the atom number of gold to reliably import them into the supposed scenario (Roca-Royes 2011, 38).

Williamson anticipates the objection and answers it as follows:

'...we need not judge that it is metaphysically necessary that gold is the element with atom number 79 before invoking the proposition that gold is the element with atom number 79 in the development of a counterfactual supposition. Rather, projecting constitutive matters such as atom

\footnotetext{
6 The process should not be described either as a byproduct of considering one arbitrary counterfactual $A \square \rightarrow C$ (Kroedel 2012, 6). I do not see how this could lead us to methodically checking for contradictions.
} 
numbers into counterfactual suppositions is part of our general way of assessing counterfactuals. The judgment of metaphysical necessity originates as the output of a procedure of that kind; it is not an independently generated input.'(Williamson 2007, 170, m.e.)

According to Williamson, a normal way of coming to know GOLD is the following: we know that gold has atom number 79, but we do not yet know GOLD. Still a reliable disposition to hold fixed 'constitutive matters' like the atom number of gold is part of our normally developed capacity of assessing relevant counterfactuals like GOLDCC. This disposition ensures that supposing gold to have a different atom number leads to a contradiction.

To get a better idea of the resulting implicit role of metaphysical constraints, consider Williamson's account of the folk physics backing our everyday counterfactual assessments:

'...the folk physics needed to derive the consequents of counterfactuals such as [If the bush had not been there, the rock would have ended in the lake] from their antecedents may be stored in the form of some analogue mechanism, perhaps embodied in a connectionist network, which the subject cannot articulate in propositional form... the supposed premises may not be stored in a form that permits the normal range of inferential interactions with other beliefs, even at an unconscious level.'(Williamson 2007, 145)

As illustrated by the role of folk physics, counterfactual reasoning recruits information that is not always readily and explicitly available to other reasoning processes. Rather it takes suitable cues for that information to play an epistemic role. A counterfactual supposition provides such cues. It also manifests our disposition to hold fixed constitutive matters like the atom number of gold. The disposition may be tacit; we do not have to be aware of it independently of manifesting it. 
Williamson's reply also provides resources for defending conceivability-based modal epistemology. ${ }^{7}$ It can be added to the common ground with a convincing conceivability-based approach: the modal inconceivability of gold having a different atom number depends on our implicit dispositions to keep constitutive matters in using imagination to address modal issues.

\subsubsection{Cognitive Dissonance as an Operational Criterion}

I elaborate one exemplary way of spelling out the psychological immediacy of modal reasoning: the experience of irremediable cognitive dissonance provides an operational criterion of impossibility.

According to Williamson, the process of developing relevant counterfactuals is largely immediate, i.e. we do not explicitly and attentively reason from outright modal premises like 'atom number is necessary' to relevant CC like GOLDCC. There is an independent argument why not only our assessment of relevant $\mathrm{CC}$ but the whole process of proceeding from a modal question via a counterfactual supposition to a modal claim must often be immediate. Jago (forthcoming) argues against imagination-deploying accounts that people have modal knowledge without knowing the relationship between imaginability and possibility, some pertinent $\mathrm{CC}$, or Williamson's equivalences. A principled answer to Jago's observation is that these things do not have to be believed or known but only have to form part of a reliable method of belief formation,

\footnotetext{
${ }^{7}$ It may even provide resources for an internalist justification, raising a reflective awareness of why imagining is suitably constrained (cf. Vaidya and Wallner forthcoming).
} 
comparably to how Williamson describes the role of folk physics (Williamson 2007, 146, cf. YliVakkuri 2013, 619, Strohminger and Yli-Vakkuri 2018, 314).

The extended immediacy claim may seem problematic, but the problem it answers to generalizes beyond imagination-deploying epistemology. For instance, Jago himself endorses an essence-based epistemology. If reasoning from essences to modal claims had to proceed via explicit knowledge of essences and bridge principles connecting essences and modality (cf. Mallozzi forthcoming), Jago's argument would backfire: some people have modal knowledge without endorsing corresponding claims to essentiality. To avoid the objection, essentialists must hold that many modal claims result from a merely implicit grasp of essences. Generalizing, any approach to modal epistemology may have to assume some immediate psychological process of reasoning from knowledge of the actual world to modal claims.

Though immediate, the process must nevertheless be influenced by explicit reasoning. First, as distinguished from some non-modal uses of imagination, it must be sensitive to the issue addressed. In Williamson's account, a modal issue must trigger the development of a counterfactual supposition. Second, the imaginative development must be sensitive to theoretical knowledge. For instance, our considerate verdict on GOLD depends on scientific knowledge about the role of atom numbers.

Taking into account these requirements, the normal use of imagination to arrive at a modal claim looks roughly as follows: addressing a modal issue whether $A$ is possible triggers an exercise of imaginatively developing a counterfactual supposition that $A$; that supposition does not have to be explicit. The development draws on any relevant knowledge. The relevant knowledge must largely inform our immediate dispositions to import certain facts into the imagined scenario. When they are imported into an impossible $A$-scenario, the scenario becomes incoherent. Becoming aware of this incoherence, we answer the possibility question in the 
negative. In contrast, if sufficient development does not lead to incoherence, we give a positive answer.

I shall illustrate the process by GOLD: we tackle the question whether the atom number of gold could be different by imaginatively developing a scenario largely in the way we would develop an explicit counterfactual supposition of gold having a different atom number. Bringing to bear our knowledge of chemistry on the scenario, we feel compelled to import the actual atom number of gold. We notice that the scenario becomes inconsistent as it is both true in the scenario that gold has a different and that it has its actual atom number. The inconsistency encountered leads us to deny GOLD.

However, heeding the lessons about immediacy, we should not expect the transition to the modal conclusion to be mediated by an explicit endorsement of GOLDCC. Our awareness of the counterfactual will often be merely implicit. We become aware of a peculiar clash between the supposition to be developed and the constraints on the imaginative development. We may explicate this clash by GOLDCC and then proceed via the logical equivalence to the modal claim. But we cannot always be expected to embark on such an explicit reasoning process. Often we simply proceed from being aware of the clash to the modal claim. The justificatory role of this process may be comparable to that of other immediate albeit reliable methods of belief formation.

What is it like to be aware of a clash between the supposition and largely implicit modal constraints? As an example for a psychological framework that promises to answer this question I shall consider constraint satisfaction theory (e.g. Shultz and Lepper 1996). A successor of the historical cognitive dissonance theory (Festinger 195s7), it sits well with Williamson's view that constitutive matters form 'implicit constraints, perhaps embodied in a connectivist network'. Here is the basic idea: 
'... dissonance is a state of tension that people are motivated to reduce. Any two cognitions are dissonant when, considered by themselves, one of them follows from the obverse of the other... How dissonance actually gets reduced depends on the resistance to change of the various relevant cognitions. Resistance derives ... from the degree to which the cognition is anchored in reality, and the difficulty of changing those aspects of reality.'(Shultz and Lepper 1996, 219)

Dissonance is triggered by incompatible cognitions. We try to reduce it by varying these cognitions, depending on which displays more resilience in virtue of being more firmly 'anchored in reality'.

In the case of counterfactual considerations, the resilience becomes modal. In our dealing with reality, different constraints on imagining counterfactual scenarios have become more or less deeply entrenched. Constraints like the atom number of gold seem to us so deeply rooted in the make-up of reality that we are disposed to uphold them at all costs, even when imagining scenarios that require us to drop them. We use a dissonance which cannot be remedied by weakening the conflicting constraints as our operational criterion of impossibility.

I offer my hypothesis of a dissonance test only as an exemplary way of fleshing out imagination-deploying modal epistemology. If such an epistemology is to work, there must be some psychological mechanism as described. I have given reasons why any modal epistemology may have to assume some immediate process like my dissonance test. While limiting my liabilities, I shall nevertheless try to support the hypothesis of a dissonance test to the best of my abilities.

\subsection{Imaginative Elaboration and Dissonance}


I explain how the dissonance test works by imaginative experimenting.

The hypothesis of a cognitive dissonance triggered by constitutive facts may be hard to swallow: there is no direct empirical evidence for it; worse, whether a scenario is more or less smooth to imagine does not seem to depend on its possibility. On the one hand, we can easily imagine impossible objects like unicorns to exist; on the other hand, we have difficulties imagining close metaphysical possibilities like matter according to quantum physics. Hence psychological phenomena like cognitive dissonance do not seem to manifest the boundaries of metaphysical possibility. ${ }^{8}$

To dispel this concern, it may help to consider a pedestrian example of the dissonance I have in mind. When can use imagination to figure out whether it is feasible to haul one particular piano (as a whole) upstairs through the narrow doorway (Byrne 2007, 136). ${ }^{9}$ Our imagining is implicitly streamlined by our grasp of the issue and our background information. Streamlining prevents us from imagining the piano disassembled, shrinking or quantum-tunneling, though we can imagine such things in principle. Only some variations are permissible. Imagining the piano carried horizontally, we realize that it does not fit. Imagining it turned by different angles, we again realize that it does not fit. Having exhausted the alternative moves, we encounter a dissonance between imagining the piano being hauled upstairs and importing streamlining conditions like keeping the relative dimensions of the piano and the doorway. We conclude that it is not feasible to haul the piano upstairs. I suggest that the dissonance which indicates that some

\footnotetext{
${ }^{8}$ This objection was raised by an anonymous referee.

${ }^{9}$ Of course there are other ways of settling such mundane issues, for instance induction from comparable situations in which a piano had to be hauled upstairs.
} 
$A$ is metaphysically impossible works in the same way save for the different constraints imposed by our grasp of the issue and our background information.

\subsubsection{A Differential Diagnosis of Dissonance}

I distinguish the phenomenon of dissonance from neighbouring ones.

I shall now give a differential diagnosis of the cognitive dissonance just illustrated. When developing an $A$-scenario to see whether $A$ is possible, we realize that the scenario has to satisfy conflicting requirements without feeling able to dissolve the conflict. This experience should be distinguished from neighbouring phenomena. It is to be distinguished from imaginative resistance as typically encountered in imagining morally repugnant scenarios. Such a resistance is sensitive to other constraints on imagining than those imposed by addressing a modal issue. It is also phenomenologically different in typically involving emotional distress.

The right kind of dissonance is also to be distinguished from other difficulties experienced in imagining. Difficulties may be independent of the use imagination is put to. For instance, they may be due to the complexity of a scenario. ${ }^{10}$ Or they may be specific to one use of imagination rather than others. Arguably there is a highly licentious use of imagination, which allows us to imagine anything we can grasp (Priest 2017). Imagining a unicorn in this way won't lead to dissonance. We do not care about the metaphysical requirements of unicorns. But there is also a more restrained use of imagination, which is triggered by properly focusing imagination on solving an issue like whether the piano can be hauled upstairs. When the issue is whether a unicorn is possible, a diligent imaginer is aware of additional metaphysical requirements coming 
with unicorns as a purported natural kind. The constraint on an imagined unicorn becomes that it belongs to a natural kind. The facts that make for its kindhood must be available for being imported into the scenario. This requirement clashes with one's knowledge that there are no unicorns, hence no facts to be imported. The imaginer becomes enmeshed in a dissonance of the right kind (unicorns have their actual deep structure vs. there is no actual deep structure).

In the case of unicorns, it may take some diligence to appreciate the requirements of kindhood. I propose to distinguish prima facie dissonance from secunda facie dissonance. When we find it difficult to modally imagine material objects according to quantum mechanics (subatomic particles as interpenetrating clouds with no determinate spatiotemporal location), the dissonance merely arises prima facie from a clash with our folk theory of material objects (cf. Spelke 1990). Once we appreciate how quantum mechanics explains and revises our folk theory, we realize that the latter must be suspended as far as it conflicts with quantum mechanics.

\subsubsection{Honing our Sensitivity by Imaginative Experiments}

I explain how imaginative experiments serve to manifest and test implicit constraints.

I shall exploit the notion of secunda facie dissonance to make the dissonance hypothesis more flexible. Especially in the case of rather abstract and theoretical constraints like atom number or kindhood, general theoretical knowledge and understanding may not yet be sufficient for developing a proper sensitivity for constitutive matters that would lead to cognitive dissonance. Rather such a sensitivity will often have to be honed by breaking down the general issue into

${ }^{10}$ Priest $(2017,191)$ gives the example of grasping an indefinitely long sentence. 
more detailed test scenarios, which enmesh one in more localized and specific experiences of dissonance. We may call this the experimental role of imagination.

I shall illustrate how such a honing works. In my piano example, we have to imagine the piano rotated around different angles to realize the dissonance between moving the piano upstairs and the circumstantial restrictions. The case of GOLD is comparable: a counterfactual supposition requires you to vary the atom number of gold. You may not immediately feel the dissonance, but you should be aware that the details are more challenging than, say, imagining the price of gold to rise. You cannot simply disregard these details 'as determinate' somehow (Yablo 1993, 28) but have to explore ways of building up a suitable variant of gold. You experience a certain leeway how to bring about the change, but also certain streamlining forces. Whenever you make a constructive move, you feel compelled to make further moves.

You may start with a minimal change $79 \pm 1$. Developing this change, you realize that the resulting atom numbers yield different elements, platinum (78) and mercury (80). At this point the development will already trigger some dissonance, provided it is part of your understanding of platinum and mercury that they are not gold. Analogously for changing the atom number by $79 \pm n$ for $n>1$. Still you may be uncertain whether some close variant of normal gold could be platinum, mercury, or some other element, or whether other variations might dissolve the dissonance. Then you have to experiment further, change the atom numbers of several elements simultaneously, change the theoretical roles of elements and their subatomic constituents, and so on. Pursuing these alternatives, you will eventually either be satisfied with your scenario or encounter an irremediable dissonance.

If imagination-deploying modal epistemologies are correct and we can know GOLD, any of your imaginative experiments should eventually lead to specific experiences of cognitive dissonance. Any concrete way of elaborating the variation clashes with some cognition. The 
frustrating experiences of ending in a predicament add to your awareness of the resilience of atom numbers and may eventually give rise to a more direct general dissonance between the request to change them and your disposition to preserve them.

I have discussed how the imaginative elaboration of concrete $A$-scenarios activates and hones our sensitivity to constitutive facts bearing on the possibility of $A$. I shall highlight one particular subtask of elaboration: tightening our grip on the precise form and scope of the resulting constraints by weighing them against test cases. Imagination is often associated with the method of cases (cf. Jackson 1998 on Example 3 in section 2.1.). Assume one wonders whether one may harm another person. One is vaguely aware of a principle of not harming others. The best way of getting a more precise grip on the principle may be test scenarios, for instance a scenario of self-defence. When we want to know whether one may harm others, such scenarios may lead to qualifying the principled moral constraint such as to be reconciled with the permission to harm others: one must not harm others except in situations of self-defence. In such situations, one may harm others. I envisage a similar role for conjuring up scenarios in the dialectics between possibility issues and relevant constraints. When some constraint seemingly precludes $A$, certain $A$-scenarios may allow us to qualify the constraint and to reconcile it with $A$. Sometimes conjuring up the right test scenarios is highly demanding. The proper functioning of imagination in doing so is part of the latter's justificatory role.

I said at the beginning that I am open to a more pluralistic or non-uniform approach. The experimental role of modal imagination as described can be combined with a conciliatory stance towards alternatives like essence-based or theory-based accounts (e.g. Fischer 2016). Sometimes explicit reasoning may lead without recruiting imagination from theories about the actual world like chemistry to their modal impact. One may even use theory to settle issues like whether atom number is essential before embarking on modal imagination or more directly proceeding to 
claims like GOLD. Still imaginative experimenting may play an indispensable role in bringing out and testing the status of constitutive matters. To ascertain that atom number is essential, we might have to play through relevant alternatives like slightly changing the theoretical roles of subatomic particles. Imagination is instrumental in creatively developing such relevant alternatives.

In sum, Williamson's view and my proposed elaboration in terms of cognitive dissonance can be made more palatable by breaking down the resilience of modal constraints like atom number to more specific and localized experiences of dissonance encountered in spelling out imaginative trials how to reconcile $A$ with the metaphysical structure of the world. In the course of elaborating, we become aware of how our theory bestows the status of 'constitutive matters' on certain facts. Alternative modal epistemologies arguably need a similar psychological process, but the experimental character of imagination seems especially amenable to it.

\section{Conceivability without Counterfactuals}

\subsection{Counterfactual Reasoning Detracts from the Experimental Use of Imagining: Some}

\section{Examples}

I present the argument against the counterfactual-based account: imaginative development as described supplants counterfactual reasoning as a guide to possibility. While imagining succinct experimental scenarios may require us to steer out into modal space, counterfactual reasoning unduly restricts our attention to closest scenarios.

I shall now argue that the modal use of imagination and counterfactual reasoning come apart. I repeat the core argument: 
(i) Let $M$ be the canonical method of acquiring modal knowledge whether $A$ is possible and $I$ be the issue whether a contradiction is true at the closest $A$-scenarios.

(ii) According to the counterfactual-based account, addressing $I$ is part of $M$.

(iii) The canonical method $\mathrm{M}$ is coherent imagining.

(iv) Addressing $I$ is not part of coherent imagining.

(v) The counterfactual-based account is false.

The argument can be introduced by coming back once more to the issue of robustness. One crucial question is in what direction to flesh out an $A$-scenario. As Williamson emphasizes, 'lines of reasoning can be continued indefinitely from any given premise.'(Williamson 2007, 153) We do not only need a sense of when a scenario is sufficiently fleshed out, but also of relevant ways of fleshing it out. I take this to be a justificatory requirement. Our imagination-based verdict must cover the relevant ways of fleshing out a scenario. While many approaches to modal imagination do not provide much guidance to what counts as relevant, Williamson's counterfactual account provides guidance: relevant ways minimally depart from actuality. However, this leads us in the wrong direction. We need to consider ways which are subjectively and objectively apt instead.

Often modal constraints on counterfactual suppositions have to be activated and tested by imaginatively exploring suitable ways of embedding $A$ (the possibility at issue) into an overall situation. These ways have to be suitable in two respects. They have to be psychologically suitable for tightening our grip on the pertinent constraints and triggering relevant dissonance, and they have to be objectively suitable in attuning those psychological features to the logical and metaphysical structure of modal space. If the right conditions are in place, i.e. we properly understand the question, have comprehensive worldly knowledge, have done sufficient 
imaginative experimenting etc., cognitive dissonance should arise precisely if $A$ violates some metaphysical constraint. Often we will need to explore a variety of ways for $A$ to be made true, but there is no principled reason why these ways have to minimally depart from actuality.

I shall corroborate my point by four possibility issues. The modal claims at stake are exemplary. I do not incur a commitment to them.

Example 1 illustrates how standard conceivability arguments for possibility come apart from the counterfactual-based approach due to the psychological requirements of making $A$ scenarios palatable to us. Here is Bohn on the possibility of junky worlds:

'Now consider the following scenario. Everything in this world is spatially extended and just one half of something else that is also spatially extended. That is, for any thing in this world, there is something else of which it is a spatial proper part. Or consider this scenario. Our universe is a miniature replica universe housed in a particle of a bigger replica universe, which is again a miniature replica universe housed in a particle of an even bigger replica universe, and so on ad infinitum. Conceiving of these scenarios amounts to conceiving of worlds in which everything is a proper part. Let's call such worlds, junky worlds. Official definition:

world $w$ is junky $=_{\mathrm{df}}$ anything in $w$ is a proper part.

Having thus conceived of junky worlds, we seem provided with some prima facie reasons to think such worlds are possible.'(Bohn 2009, 28)

Bohn does not simply ponder the counterfactual supposition of a world in which everything is a proper part; he creatively crafts two recipes for such a world, in one case putting halves together, in the other case a matryoshka doll-like encapsulation of replica universes. These recipes are neither mere adornments, nor are they the result of looking for junk-worlds closest to ours. When 
we read the definition of junk, its possibility is not obvious. It is not immediately transparent to us what the more concrete constraints on a junky world are, and whether they can be met. We need to flesh out junk-scenarios in a pedagogically convincing way. Bohn's recipes fit the task. They are crafted such as to guide fleshing out the abstract mereological requirements of junk by an easy algorithmic structure: wholes are put together from things of a single kind which display simple non-gerrymandered mereological features. Construing arguments like Bohn's as a counterfactual test detracts our attention from their crucial feature: we look first and foremost for subjectively and objectively apt ways of making a world junky, irrespectively of whether they are closest ways (for a qualification see section 2.2., Objection 1).

Example 2: Whereas my first example illustrated the pedagogical role of imagining succinct ways for making $A$ real, the function of imagining scenarios in testing constraints can be illustrated by a claim used by Roca-Royes (2011, cf. my section 1.3.2.):

(ORIGIN) Aristotle could not have originated from a different zygote than he actually came from.

Assume ORIGIN is true and knowable. According to the counterfactual account, supposing Aristotle to have originated from a different zygote conflicts with the constraint that ties Aristotle to the zygote he actually came from. Yet we may need to creatively flesh out suitable scenarios in order to properly appreciate the relevance and the scope of the metaphysical constraint. Perhaps Aristotle could have arisen from something that came close enough to the actual zygote to play the metaphysical role of the latter. To check, we may have to consider fanciful scenarios like the following: Aristotle developed not from the actual zygote but from some perfect molecule-permolecule replica implanted by advanced extra-terrestrial scientists into the body of his mother at 
the very moment of his conception. Again metaphysical considerations are relevant (cf. Rohrbaugh and DeRosset 2004), but again they might have to be supplemented by imagining test scenarios. A requirement of staying close to actuality does not help in finding such scenarios. Example 3 draws a parallel to Frank Jackson's use of the method of cases. Consider

(CAT) Cats necessarily are animals.

Jackson here is interested in questions of aprioricity, but his remarks bear on my issue:

'Our failure to decide in advance how we would jump in fantastical, remote cases gives philosophers with their notorious ability to think up fantastical, remote cases, plenty of scope to come up with a case for which it is undecided whether, as it just might be, 'cat' and 'animal' apply, and so is a case where we can be induced, without going against anything determinate in the meaning of the terms, to apply, say, 'cat' and not apply, say, 'animal'. Thus, the case becomes one where cats are not animals.'(Jackson 1998, 54)

I use the quote only to illustrate my point: modally imagining a cat that is no animal probably triggers prima facie cognitive dissonance. Cats being animals is a candidate for a constitutive fact. Yet to see whether the dissonance is irremediable, we may have to go through 'fantastic, remote cases' like perfect mechanical facsimiles of cats to test the implicit metaphysical constraints on being a cat. Again the real work of imagination consists not in drawing consequences from the supposition of a cat which is no animal but in creatively finding a way for a cat not to be an animal. 
Example 4 elaborates on this parallel to the method of cases. It is taken from Williamson's formalization of Gettier reasoning (2007, 183-195). For some particular Gettier case $G C$ :

(G1) Necessarily, for any subject $S$ and proposition $P, S$ knows $P$ if and only if $S$ has justified true belief in $P$.

(G2) Possibly, some $S$ stands to some $P$ in the relation described by $G C$.

(G3) If some $S$ were related to a proposition $P$ as described by $G C, S$ would have justified true belief in $P$ without knowing $P$.

(G4) It is possible that some $S$ has a justified true belief in some $P$ without knowing $P$. Thus: $\operatorname{not}(\mathrm{G} 1)$.

Williamson's construal is appealing if we start from (G1) as the claim to be refuted and immediately come up with a counterexample (as Gettier 1963 did). But consider a reasoner pondering (G4) without having (G1) in mind. Is it possible that someone has a justified true belief without knowing $(J T B N K)$ ? Applying Williamson's theory, the reasoner should develop a counterfactual supposition à la 'some $S$ has $J T B N K$ ' to see whether it entails a contradiction. Assuming (G1) is false, the development won't yield a contradiction. But how precisely is the reasoner to proceed in developing the supposition?

It seems highly likely that Gettier-style examples will play a role even in directly addressing the possibility claim (G4). If the theory that knowledge is justified true belief (JTB) has any plausibility, the connection knowledge-JTB is a salient candidate for a binding constraint on counterfactual reasoning, comparable to the connection element-atom number. Modally imagining that some $S$ has $J T B N K$ is likely to trigger prima facie dissonance. Part of dissolving the latter is to look for a suitable way to embed the imagination that someone has JTBNK into a 
richer scenario. The richer scenario should be hospitable to JTBNK; it should uncover eligible ways of implementing JTBNK. Gettier cases fit the task. We accept (G4) if we have sufficiently developed a Gettier scenario in which someone has JTBNK. To be sure, Gettier cases may represent the closest ways of having JTBNK. Yet the counterfactual-based account does not provide guidance as to how we methodically arrive at them.

\subsection{Coherent Imagining vs. Counterfactual Reasoning}

Coherent imagining stands on its own as a direct guide to modal knowledge, while counterfactual reasoning would amount to an unnecessary detour.

Taking stock, using imagination to answer whether $A$ is possible requires us to embed $A$ into some suitable way for $A$ to come about. I shall call the procedure of finding and elaborating such an embedding coherent imagining:

The use of imagination to answer whether $A$ is possible consists in elaborating concrete $A$ scenarios in ways that are suited for us to become aware of relevant constitutive facts and any dissonance encountered by combining them with A. If imagination properly responds to the issue, it is constrained by such facts. When one encounters a dissonance, one imaginatively explores ways of dissolving it. Depending on whether the attempt of finding such ways robustly succeeds or fails, one judges A possible or not.

Coherent imagining is not subject to minimum alteration. Still it may be the most effective way of figuring out relevant counterfactuals $C C$. We use coherent imagining and then 
take an additional step: if coherent imagining uncovers some way of avoiding any clash between the pertinent constraints and the supposition, it thereby provides a candidate for the antecedent situation closest to actuality, though under the caveat that there may be closer ones. If there is no such way to be uncovered, there is no closest way either.

However, the consequence is that counterfactual reasoning provides no guidance to coherent imagining but rather the other way round. Coherent imagining stands on its own but counterfactual reasoning cannot. We directly approach the task of finding a coherent $A$-scenario. We thereby gather all the evidence we can get for or against both the relevant $C C$ s and the possibility of $A$. If the counterfactual test is of any avail, our failure or success in coherently imagining $A$ already tells us whether $A$ is possible. Our judgement whether $A$ is possible or not depends on our robustly encountering or not encountering cognitive dissonance in trying to imagine succinct $A$-scenarios. We do not have to draw consequences for relevant $C C$ to arrive at a modal claim. ${ }^{11}$

\subsection{Some Objections}

I elaborate on the account by answering four objections: first, in focusing on easily accessible rather than closest scenarios, coherent imagining better accommodates moderate scepticism than counterfactual reasoning. Second, coherent imagining better covers the full range of relevant

${ }^{11}$ One may object that this just is Williamson's account as interpreted by Strohminger and YliVakkuri $(2018,314)$. However, the result is that $C C$ drop out of the picture. They do not have to play any role either in formulating the account or reasoning according to it. We get just the old conceivability-based approach. 
scenarios. Third, coherent imagining does not have to be framed as counterfactual reasoning. Fourth, its advantages transfer to other epistemologies and semantics for counterfactuals.

Objection 1: Our epistemic capacities may be reliable only in dealing with scenarios that are sufficiently close to actuality (Williamson 2007, 164). First, our epistemic capacities are evolutionarily adapted to the actual world and not to far-fetched ones. Second, we always have to start from knowledge of actual facts. Yet the more far-fetched a scenario is, the less guidance actual facts provide to fleshing it out by simply importing them (Strohminger and Yli-Vakkuri 2018, 317). Williamson wants his account to be reconcilable with a moderate skepticism resulting from such caveats. My alternative does not live up to this requirement. Coherent imagination invites us to consider arbitrarily far-fetched $A$-scenarios as far as these scenarios seem hospitable to $A .^{12}$

In reply, the concern can even be used to further support a conceivability-based account as contrasted to the counterfactual-based one. Heeding doubts about our capacity to assess farfetched scenarios, we should take care to select only $A$-scenarios which we are in a good position to assess. Yet rather than considering a counterfactual, the direct way of tackling moderate scepticism is to look for easily accessible $A$-scenarios. This is part of what makes them psychologically apt for modalizing. Closeness may play a role, but only as far as nearby scenarios happen to be more easily accessible and not because we are bound to consider $A$ -

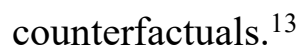

\footnotetext{
${ }^{12}$ Thanks to an anonymous referee for raising this issue.

${ }^{13}$ Some concern remains: how can we be confident that no scenario that reconciles $A$ with the pertinent constraints can be found if we have to concentrate on the easily accessible ones? This
} 
There are reasons why closest $A$-scenarios may sometimes be more difficult to access than somewhat more far-fetched ones. I illustrate the claim by the possibility of gunk, which is the opposite of junk: anything has proper parts. Strohminger and Yli-Vakkuri $(2018,317)$ mention gunk as an example for a far-fetched possibility which is difficult to assess. However, the actual world might turn out to be a gunk-world (Sider 1993, 287, Schaffer 2003, 500-506). Moreover, the infinite layers of its particular gunky microstructure may be so complicated and difficult to access that we are better at assessing the possibility of certain 'dull' and repetitive gunk-worlds comparable to the junk-worlds selected by Bohn. Albeit less similar to actuality, the dull worlds better suit our capacities. Moreover, they do not presuppose knowledge of the actual gunkstructure. Whereas the counterfactual-based approach would draw our attention to the complicated gunk-worlds close to actuality, however difficult they might be to access, a conceivability-based one always allows us to privilege the easily accessible ones. This seems preferable in addressing whether gunk is possible, regardless of whether we can eventually solve the issue.

Objection 2: Coherent imagination addresses the possibility of $A$ by embedding $A$ into a richer scenario $W_{A}$ that is hospitable to $A$. Why not rephrase the counterfactual test by replacing $\neg(A \square \rightarrow \perp)$ with $\neg\left(W_{A} \square \rightarrow \perp\right)$ ?

In reply, our justification for a modal claim depends on our sensitivity for considering the right scenarios. A counterfactual test as considered covers only part of the justificatory procedure. It ignores the essential task of manifesting and weighing the pertinent constraints by a set of

concern also applies to Williamson's original requirement of robustness. It is accommodated by the proviso that our judgement is defeasible. 
relevant scenarios, $W_{A}$ being just one of them. The conceivability-based epistemology I have in mind also accounts for this task. The task goes beyond a context of discovery as our justification depends on our sensitivity to relevant scenarios (replacing Williamson's requirement of robustness for the counterfactual development).

Objection 3: I have argued that coherent imagining can stand on its own. One may retort that the step via addressing $C C$ is inevitable. I shall consider three arguments.

First, one may insist that pondering counterfactuals is a prerequisite of grasping the modality at issue. However, we immediately understand concrete possibility issues like hauling the piano upstairs. Metaphysical possibility is often described as 'absolute', 'tout court' as contrasted to circumstantially restricted possibility (van Inwagen 1998, 72, Hale 2003, 3, Williamson 2017, 417). It seems that we can arrive at an understanding of metaphysical possibility by detaching from contextual constraints (cf. Vetter 2016).

Second, one may argue that the merely psychological phenomenon of cognitive dissonance has to be interpreted by explicit $C C$ to become evidentially relevant. However, by Williamson's own lights, it is sufficient that our modal judgement is backed by a process that is reliably constrained. To Williamson, a suitable exercise of imagination amounts to such a process. It is not in need of interpretation.

Third, imagination is not always bound to modal truth. Only counterfactuals, one may say, provide the right context for coherent imagining. Yet there is no reason why an explicit modal question should not activate coherent imagining as effectively as a counterfactual question. The question 'is it possible that $A$ ?' creates a context for coherent imagining. 
Objection 4: I followed Williamson's view concerning features like the use of imagination and minimal alteration. What if counterfactual reasoning functions differently?

In reply, coherent imagining remains a highly eligible alternative of how we form modal judgements. My exemplary discussion has revealed a general concern: the evaluation of counterfactuals is likely to come with additional requirements which detract from the bare question of possibility. There is always the alternative of using coherent imagining in more directly addressing a modal issue.

\subsection{A Parallel to Thought-Experiments}

I draw a parallel between Williamson's counterfactual-based modal epistemology and his formalization of thought-experiments. In both cases, the requirement of staying maximally close to actuality leads to difficulties, which can be avoided by an account which separates the use of imagination from counterfactual reasoning.

I shall once more come back to Example 4, drawing a further parallel to Williamson's account of thought-experiments. The parallel supports my claim that the requirements of assessing counterfactuals are orthogonal to the requirements of tackling a possibility issue. Williamson construes Gettier reasoning (G1)-(G4) via the counterfactual:

(G3) If some $S$ were related to a proposition $P$ as described by $G C, S$ would have justified true belief in $P$ without knowing $p$. 
This counterfactual-based account is faced with the so-called problem of deviant realizations. Take some particular Gettier story (cf. Williamson 2009, 467):

$G C$ : At 8:28, somebody looks at a clock to see what time it is. The clock is broken; it stopped exactly twenty-four hours previously. The subject believes, on the basis of the clock's reading, that it is 8:28.

The aim of Gettier experiments is to provide a $J T B N K$-scenario. Intuitively, $G C$ describes such a scenario. But $G C$ is a highly unspecific description, which is true of very different possible scenarios. In some of them, the subject either has knowledge or fails to be justified. To obtain such a scenario, just add the following clause to $G C$ :

$G C^{*}$ : ...The subject knew in advance that the clock had stopped exactly twenty-four hours previously.

The subject in $G C^{*}$, knowing just that the clock stopped exactly twenty-four hours earlier, can use the broken clock to know the time.

Williamson originally introduced the problem in order to motivate his formalization of thought-experiments via (G1)-(G4). However, as Williamson's critics have emphasized, (G3) is also threatened by deviant realizations (Ichikawa 2009, Malmgren 2011). If some $G C$-scenario which minimally departs from actuality is a deviant $\left(G C^{*}\right)$ scenario, the counterfactual (G3) may be false. But the thought-experiment does not seem to be vulnerable to deviant realizations in the way the counterfactual is. We dismiss deviant realizations as 'not meant' (Malmgren 2011, 279). 
Of course, we are aware that the scenario may be easily fixed or replaced by a different one. But the point pressed by Williamson's critics is that it simply does not matter what the $G C$ scenarios closest to actuality are like. We only care whether some $G C$-scenario is a scenario in which someone has justified true belief without knowledge.

I draw a parallel to the epistemology of metaphysical modality. Just as the use of counterfactuals detracts from directly addressing the issue whether $A$ is possible by coherent imagining, it seems to detract from directly addressing a thought-experimental scenario. ${ }^{14}$ The direct way is not vulnerable to deviant realizations. I have argued that the direct way to asses a possibility issue is coherent imagining. When we address the issue whether ((G4) there could be a case of $J T B N K$ ), we imagine Gettier scenarios like $G C$ which are hospitable to $J T B N K$. We do not care about fleshing out these scenarios in a way that stays closest to actuality. One may still ask how we ascertain that the scenarios imagined can be realized non-deviantly. An answer in the spirit of Williamson is that we can tell when an imagination has been sufficiently developed, though our verdict is defeasible (cf. my section 1.1., Williamson 2007, 153-155). I shall not draw consequences for the formalization of thought-experiments. In any case, our dismissal of deviant realizations might be explained by the role of coherent imagining in thought-experimental reasoning.

${ }^{14}$ Deviant realizations are also a challenge for Kroedel's $(2012,11,2017,191)$ suggestion to replace Williamson's equivalences by one particular might-counterfactual, using the equivalence $\diamond A \equiv \exists B(B \diamond \rightarrow A)$. Kroedel $(2012,9-11,2017,190-191)$ also discusses whether to use the equivalence $\square A \equiv \forall B(B \square \rightarrow A)$ instead of Williamson's, perhaps restricted to possible $B$. However, surveying all possible $B$ seems overdemanding. 


\section{Creativity and Restraint: Coherent Imagining as a Guide to Possibility}

I draw a lesson: a convincing conceivability-based approach should take on board Williamson's holistic view of properly restrained imagination, yet it should also make room for the creative role of the latter, which requires us to detach from counterfactual reasoning.

I shall close with sketching two lessons about the role of coherent imagining in figuring out modal truth. My first lesson concerns what a conceivability-based account can learn from Williamson's. Imagination is seen as a capacity of using any factual information and any other epistemic capacity 'off-line' to simulate some reality. The use of imagination pervades everyday reasoning. The resulting account supports anti-exceptionalism about modal reasoning. It is not immediately committed to invoking specific cognitive capacities or sources of knowledge like conceptual knowledge or knowledge of essences but does not exclude their use either.

The claim that imagination is properly constrained naturally follows from such an account. Williamson emphasizes that, on pain of circularity, the pertinent constraints have to be partly implicit. They are not imposed by explicit stipulation but have to be activated by suitable cues. We get an answer to complaints that modal imagination is caught in a circle or that it is not properly constrained. Of course, this answer will have to be tested against diverging accounts of imagination. But it forms part of an attractive non-sceptical approach to the cognitive role of imagination. Such an approach should also be reconcilable with less restricted uses of imagination, though.

My second lesson concerns where a conceivability-based account should part ways with a counterfactual-based one. Imagination simulates some reality. The reality simulated may substantially diverge from actuality. It takes exploring and experimenting to find the right 
scenarios. Thus, imagination works between the two poles of creativity and restraint. Williamson plausibly accounts for the aspect of restraint. I have highlighted the creative aspect.

This role of creativity further corroborates an imagination-deploying approach to modal epistemology. Imagination traditionally has been associated with creativity. There are psychological results on games of pretense confirming this view (cf. Nichols and Stich 2000, 119). In largely unrestrained pretense games, the creative use of imagination is most obvious. In modal imagination, creativity must be focused by the task of looking for a way to manifest and jointly satisfy the constraints on which the possibility of $A$ depends.

In conclusion, I have argued that imagination has a function of its own in modal reasoning, which differs from Williamson's counterfactual-based account. Still Williamson's view of imagination provides important guidance towards developing a convincing view of coherent imagination as a guide to modal truth.

Berto, F. et al. 2018. Williamson on Counterpossibles, Journal of Philosophical Logic 47. 26972715.

Berto, F., Schoonen, T. 2018. Conceivability and Possibility. Some Dilemmas for Humeans. Synthese 195, 2695-2715.

Bohn, E. D. 2009. An argument against the necessity of unrestricted composition. Analysis 69, $27-31$

Bueno, O. Shalkowski, S.A. 2015. Modalism and Theoretical Virtues: Toward an Epistemology of Modality. Philosophical Studies 172, 671-689. 
Byrne, A. 2007. Possibility and Imagination. Philosophical Perspectives 21, 125-144.

Chalmers, D. 2002. Does Conceivability Entail Possibility?. In Gendler, T.S., Hawthorne, J. (eds.), Conceivability and Possibility, 71-125. Oxford: Clarendon Press.

Festinger, L. 1957. A Theory of Cognitive Dissonance. Stanford, CA: Stanford University Press.

Fiocco, M.O. 2007. Conceivability, Imagination and Modal Knowledge. Philosophy and Phenomenological Research 74, 364-380.

Fischer, R.W. 2016. A Theory-Based Epistemology of Modality. Canadian Journal of Philosophy 46, 228-247.

Fischer, R.W., Leon, F. (eds.) 2017. Modal Epistemology After Rationalism. Dordrecht: Synthese Library

Geirsson, H. 2005. Conceivability and Defeasible Modal Justification. Philosophical Studies 122, 279-304.

Gettier, J. 1963. Is Justified True Belief Knowledge?. Analysis 23, 121-123.

Gregory, D. 2004. Imagining possibilities. Philosophy and Phenomenological Research 69, 327348. 
Gregory, D 2017. Counterfactual Reasoning and Knowledge of Possibilities. Philosophical Studies 174, 821-835.

Hale, B. 2003. Knowledge of Possibility and of Necessity. Proceedings of the Aristotelian Society 103, 1-20.

Hill, C. 2006. Modality, Modal Epistemology, and the Metaphysics of Consciousness. In Nichols, S. (ed.), The Architecture of the Imagination, 205-235. Oxford: Oxford University Press.

Ichikawa, J. 2009. Knowing the Intuition and Knowing the Counterfactual. Philosophical Studies $145,435-443$.

Ichikawa, J., Jarvis, B. 2012. Rational Imagination and Modal Knowledge. Nô̂s 46, 127-158.

Jackson, F. 1998. From Metaphysics to Ethics. Oxford: OUP.

Jago, M. forthcoming. Knowing How Things Might Have Been. Synthese.

Kment, B. 2014. Modality and Explanatory Reasoning. Oxford: Oxford University Press.

Kment, B. forthcoming. Essence and Modal Knowledge. Synthese.

Kripke, S. 1980. Naming and Necessity. Cambridge, Mass.: Harvard University Press. 
Kroedel, T. 2012. Counterfactuals and the Epistemology of Modality. Philosophers' Imprint 12, $1-14$.

Kroedel, T. 2017. Modal Knowledge, Evolution, and Counterfactuals. In Fischer, R.W., Leon, F. (eds.), Modal Epistemology After Rationalism, 179-196. Dordrecht: Synthese Library.

Kung, P. 2016. You Really Do Imagine It: Against Error Theories of Imagination. Noûs 50, 90120.

Lam, 2018. Is Imagination too Liberal for Modal Epistemology?. Synthese 2018, 195, 21552174.

Lange, M. 2005. A Counterfactual Analysis of the Concepts of Logical Truth and Necessity. Philosophical Studies 125, 277-303.

Mallozzi, A. forthcoming. Putting Modal Metaphysics First. Synthese.

Malmgren, A.S. 2011. Rationalism and the Content of Intuitive Judgements. Mind 120, 263-327.

Martínez, M. 2015. Modalizing Mechanisms. The Journal of Philosophy 92, 658-70.

Nichols, S., Stich, S. 2000. A Cognitive Theory of Pretense. Cognition 74, 115-147. 
Peacocke, C. 1999. Being Known. Oxford: Clarendon Press.

Priest, G. 2017. Thinking the Impossible. Argumenta 2, 181-194.

Roca-Royes, S. 2011. Conceivability and De Re Modal Knowledge. Noûs 45, 22-49.

Rohrbaugh, G., DeRosset, L. 2004. A New Route to the Necessity of Origin. Mind 113, 705-725.

Schaffer, J. 2003. Is There a Fundamental Level?. Noûs 37, 498-517.

Shultz, T.R., Lepper, M.R. 1996. Cognitive Dissonance Reduction as Constraint Satisfaction. Psychological Review 103, 219-240.

Sider, T. 1993. Van Inwagen and the possibility of gunk. Analysis 53, 285-289.

Spelke, E. 1990. Principles of Object Perception. Cognitive Science 14, 29-56.

Strohminger, M., Yli-Vakkuri, J. 2018. Moderate Modal Skepticism. In Benton, M., et al. (eds.), Knowledge, Belief, and God: New Insights in Religious Epistemology, 302-321. Oxford: Oxford University Press.

Tidman, P. 1994. Conceivability as a test for possibility. American Philosophical Quarterly 31, 297-309. 
Vaidya, A., Wallner, M. forthcoming. The Epistemology of Modality and the Problem of Modal Epistemic Friction. Synthese.

Van Inwagen, P. 1998. Modal Epistemology. Philosophical Studies 92, 67-84.

Vetter, B. 2016. Williamsonian Modal Epistemology, Possibility-Based. Canadian Journal of Philosophy 46, 766-795.

Williamson, T. 2007. The Philosophy of Philosophy. Oxford: Blackwell.

Williamson, T. 2009. Replies to Ichikawa, Martin and Weinberg. Philosophical Studies 145, 465476.

Williamson, T. 2017. Modal Science. Res Philosophica 94, 415-436.

Yablo, S. 1993. Is Conceivability a Guide to Possibility?. Philosophy and Phenomenological Research 53, 1-42.

Yli-Vakkuri, J. 2013. Modal Knowledge and Counterfactual Knowledge. Philosophical Studies $162,605-623$. 